\title{
Simulational analysis of two controllers for variable speed limit control
}

Edouard Ivanjko PhD

Associate Professor, Faculty of Transport and Traffic Sciences, University of Zagreb, Zagreb, Croatia (Orcid:0000-0002-2083-0672)

\section{Krešimir Kušić MEng}

Research Assistant, Faculty of Transport and Traffic Sciences, University of

Zagreb, Zagreb, Croatia (corresponding author: kresimir.kusic@fpz.hr)

(Orcid:0000-0001-9244-2364)

\author{
Martin Gregurić PhD \\ Postdoc, Faculty of Transport and Traffic Sciences, University of Zagreb, \\ Zagreb, Croatia (Orcid:0000-0003-0100-7127)
}

Variable speed limit control (VSLC) is one of the services from the domain of intelligent transport systems applied to alleviate or prevent congestion on urban motorways. The main idea of VSLC is to change the speed limit according to current traffic or weather in order to improve the traffic situation. Improvement of the traffic situation on urban motorways depends on the applied controller and its parameters. For this reason, the controllers chosen for implementation have to be tested in simulations using a realistic urban motorway segment, placement of sensors and traffic scenario, and a relevant set of control parameters. An analysis of two VSLC controllers, including the influence of their parameters on controller performance with respect to traffic and environmental aspects, is presented in this paper. To this end, an appropriate microscopic simulation framework was developed, the fundamental diagram of a simulated synthetic urban motorway segment was measured and traffic parameters, with vehicle emissions values, were obtained. The effectiveness of the two analysed controllers was compared based on the obtained simulation results. The results revealed that appropriate settings of the controller are crucial to ensure optimal use of urban motorways with minimum travel times and vehicle emissions.

$\begin{array}{llll}\text { Notation } & & T_{0} & \text { simulation sampling time } \\ C_{i} & \text { control variable associated with each } & T_{1} & \text { control time steps } \\ & \text { motorway section }\left(i \in I_{\mathrm{v}}\right) & V_{\mathrm{c}} & \text { critical speed } \\ C_{\mathrm{v}} & \text { positive constant } & V_{i}\left(k T_{1}\right) & \text { final speed limit for motorway section } i \\ f\left(Q_{i}\left(k T_{i}\right)\right) & \text { mapping function } & \left.V_{i}(k-1) T_{1}\right) & \text { speed limit from previous time step } \\ I_{\mathrm{r}, i} & \text { set of downstream sections relevant to section } i & \bar{V}_{i}\left(k T_{i}\right) & \text { speed limit from controller } \\ I_{\mathrm{V}} & \text { set of road sections in which speed limits } & V_{\mathrm{max}} & \text { maximum allowed speed limit } \\ & \text { are controlled } & V_{\text {min }} & \text { minimum allowed speed limit } \\ K_{\mathrm{r}} & \text { control parameter } & v & \text { average speed } \\ K_{\mathrm{V}} & \text { controller gain parameter } & \bar{v}_{i}\left(k T_{0}\right) & \text { average speed at section } i \text { at time } k T_{0} \\ k & \text { time step } & \bar{v}_{\text {in }}\left(k T_{0}\right) & \text { average speed at the beginning of section } i \\ l_{i} & \text { length of section } i & \bar{v}_{\text {out }}\left(k T_{0}\right) & \text { average speed at the end of section } i \\ N_{\mathrm{C}} & \text { positive integer } & \Delta_{+} & \text {activation design parameter } \\ N_{\mathrm{r}, i} & \text { number of sections in the set } I_{\mathrm{r}, i} & \Delta_{-} & \text {deactivation design parameter } \\ n_{i} & \text { number of vehicles passing through motorway } & \rho_{\mathrm{c}} & \text { critical density } \\ & \text { section } i & \rho_{\mathrm{d}} & \text { desired density } \\ n_{\mathrm{in} i}\left(k T_{0}\right) & \text { number of incoming vehicles, regarding } & \rho_{i}\left(k T_{0}\right) & \text { current density in section } i \text { at time } k T_{0} \\ & \text { section } i & \rho_{i}\left((k-1) T_{0}\right) & \text { density from previous time step } \\ n_{\text {out }}\left(k T_{0}\right) & \text { number of outgoing vehicles, regarding } & \rho_{i+1}\left((k-1) T_{1}\right) & \text { density in downstream section from previous } \\ & \text { section } i & & \text { time step } \\ O\left(k T_{1}\right) & \text { measured downstream occupancy at time } k T_{1} & \rho_{i+1}\left(k T_{i}\right) & \text { density in downstream section at time } k T_{1} \\ O_{\mathrm{c}} & \text { critical occupancy } & \rho_{j}\left(k T_{1}\right) & \text { density of relevant downstream motorway } \\ O_{\mathrm{d}} & \text { desired downstream occupancy } & & \text { sections in time step } k T_{1} \text {, regarding section } i \\ Q_{i}((k-1) & & \end{array}$

$Q_{i}\left((k-1) T_{1}\right) \quad$ flow from previous time step

$Q_{i}\left(k T_{0}\right) \quad$ traffic flow across section $i$ at time $k T_{0}$

$Q_{i}\left(k T_{1}\right) \quad$ metered flow rate at time $k T_{1}$

$Q_{\max } \quad$ maximum traffic flow achieved around speed $V_{\mathrm{C}}$

$Q_{\text {min }} \quad$ flow corresponding to $V_{\text {min }}$

$R\left((k-1) T_{1}\right) \quad$ computed on-ramp flow in previous time step

$R\left(k T_{1}\right) \quad$ computed on-ramp flow at time $k T_{1}$

\section{Introduction}

Motorways are built to ensure a high level of service (LoS) for road users. A special case of motorways are urban motorways near densely populated areas. They are used as bypasses around larger cities and to connect suburbs with the city centre and different areas in large conglomerations. Urban motorways are characterised by a larger number of closely located 
on- and off-ramps to serve local and transit traffic. They are placed where traffic congestions occur daily (Roess et al., 2011). The reason for this congestion is the interaction of the motorway's main traffic flow with the input flow from onramps and output flow related to off-ramps. Additionally, congestion spillback from the local urban traffic network can occur throughout the off-ramp, creating congestion on the motorway (Falcocchio and Levinson, 2015).

Today's traffic-control services from the domain of intelligent transportation systems are applied to alleviate or prevent congestion on urban motorways. Their goal is to raise the LoS of urban motorways, increase traffic safety and reduce vehicle emissions ( $\mathrm{Li}$ and Ranjitkar, 2015; Rämä and Schirokoff, 2004). Ramp metering and variable speed limit control (VSLC) are commonly used, and these two traffic-control services can also be used together in coordination. The focus of this paper is on VSLC.

The main idea of VSLC is to change the current speed limit according to the current traffic or weather situation by using variable message signs (VMSs) to inform drivers (Khondaker and Kattan, 2015). Therefore, an appropriately set speed limit can increase traffic throughput and safety and reduce the measures of effectiveness (MoE), evaluated here using total time spent (TTS), travel time (TT) and vehicle emissions. For these reasons, VSLC is often used on urban motorways, taking into account the influence of weather and traffic parameters separately or simultaneously (Li and Ranjitkar, 2015; Rämä and Schirokoff, 2004). Only the influence of different traffic conditions is considered for analysis in this paper.

Research related to VSLC has been very active over the last 30 years. The review by Khondaker and Kattan (2015) describes the approaches that have been applied mostly for VSLC, starting from simple rule-based approaches and continuing to more advanced strategies based on the prediction of traffic states. However, the drawback of this review is that the given approaches are not implemented and compared by applying an appropriate use case. Sadat and Celikoglu (2017) evaluated a logic decision-tree-based controller for VSLC in microscopic simulations, but no detailed analysis of the influence of the controller's parameters was provided. A similar situation was covered by Müller et al. (2015), who analysed the influence of the placement of VMSs and the length of the area under VSLC in several scenarios. Both papers emphasise the application of microscopic simulators to simulate traffic processes on urban motorways accurately. In the work described in this paper, microscopic simulations were also used to make a detailed analysis of two chosen VSLC controllers according to traffic and vehicle emission indicators, including the influence of controller parameters. Vehicle emissions are very important, given the EU's plans to significantly reduce greenhouse gas (GHG) emissions produced by the EU transport sector (EC, 2011). Emissions from the road transport sector are responsible for $72 \%$ of total EU GHGs from the transport sector, and they mostly affect densely populated urban areas (EEA, 2018). For this reason, every analysis of traffic-control approaches has to consider environmental aspects.

The work presented in this paper is a continuation of previous work conducted by the authors (Gregurić et al., 2016; Kušić et al., 2016). In the paper by Gregurić et al. (2016), a short review is provided for the choice of reactive controllers for VSLC for the comparison performed by Kušić et al. (2016). However, the comparison presented by Kušić et al. (2016) contains only basic analysis regarding traffic and emission parameters without detailed analysis of the influence of controller parameters. Additionally, the flow-speed mapping function used in one of the analysed controllers was not adapted to the traffic characteristics of the used microscopic simulator (Vissim). This is now included in this paper, with details of how to obtain the fundamental diagram and traffic parameters of the controlled motorway segment in the used simulator. Added sections are important since published analyses of VSLC controllers (e.g. Carlson et al., 2010; Li and Ranjitkar, 2015) do not emphasise this issue, which is interesting for both research and for practical implementation.

\section{Motivation for VSLC}

When designing a method or controller for traffic control, it is imperative that each control action keeps the traffic density near the critical density. This is important because the highest traffic throughput is achieved at the critical density. If the current traffic density exceeds the critical one, capacity drop occurs and congestion starts to build up. Congestion can induce complete traffic standstill or one of two types of stopand-go traffic situations (Papageorgiou et al., 2008). The first type of stop-and-go traffic is characterised by a strong fluctuation of flow, density and speed. The second type keeps the traffic flow at an almost constant value and fluctuations are only present in speed and density (Gregurić et al., 2016). Both types of stop-and-go traffic situations produce fluctuations in speed. VSLC is applied in such traffic situations to induce homogenisation of traffic flow speeds. In such a case, VSLC has the main goal of improving the performance of traffic flows on motorways. The actions of VSLC also result in an increase in traffic safety and decreases in air and noise pollution, and induce a reduction of the impact of shockwave propagation on the mainstream (Papageorgiou et al., 2008; Rämä and Schirokoff, 2004). The latter is relevant since shockwaves in any form are present in every traffic congestion. Another important feature of VSLC applications is the reduction of the mean speed at densities below the critical density. Namely, when applied, VSLC changes the fundamental diagram of the controlled motorway section, as shown in Figure 1. The critical density is increased by changing the slope of the left-hand part of the fundamental diagram by applying different variable speed limits (Papageorgiou et al., 2008). This part of the fundamental diagram is related to 


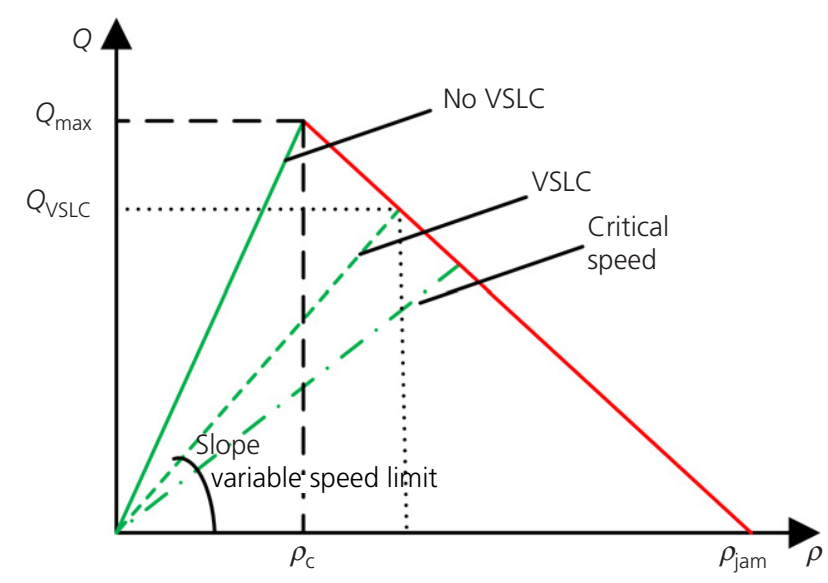

Figure 1. Fundamental diagram with and without applied VSLC (Papageorgiou et al., 2008)

stable traffic flow and presents traffic situations in which VSLC can make an improvement.

Speed reduction and homogenisation of speeds can also reduce the number of traffic accidents, which are caused by abnormalities in interactions between vehicles. Rämä and Schirokoff (2004) showed that VSLC enables a reduction in traffic accidents by up to $30 \%$. Traffic accidents can occur on motorways due to bad weather and poor road conditions. Bad weather can cause reduced visibility for drivers, while poor road conditions such as slush, wet pavements and ice can lead to fatal accidents in combination with higher speeds. Thus, in these conditions, it is favourable to reduce vehicle speeds by applying VSLC. In such cases, information about speed reductions is usually accompanied by additional information about the state of the road and/or weather. Studies conducted in Finland showed that motorways with VSLC, even though VSLC was firstly used to improve the throughput of the motorways, have increased traffic safety in cases of bad road and weather conditions (Rämä and Schirokoff, 2004).

\section{Controllers chosen for analysis}

Two reactive controllers, based on the negative feedback loop control framework, were chosen for analysis using microscopic simulations (Kušić et al., 2016). Both are based on the mapping of speed values using the fundamental flow-density correlation and are described in more detail in the following. It is worth noting here that, to apply VSLC, a controlled motorway segment must be divided into $N$ sections.

\subsection{The mainline virtual metering (MVM) controller}

The MVM controller uses the flow-speed relationship to map the ramp metering rate into speed limits. It is based on the principle of ramp metering that, in general, increases the throughput of an urban motorway by limiting the on-ramp flow into the motorway mainstream flow. The same principle

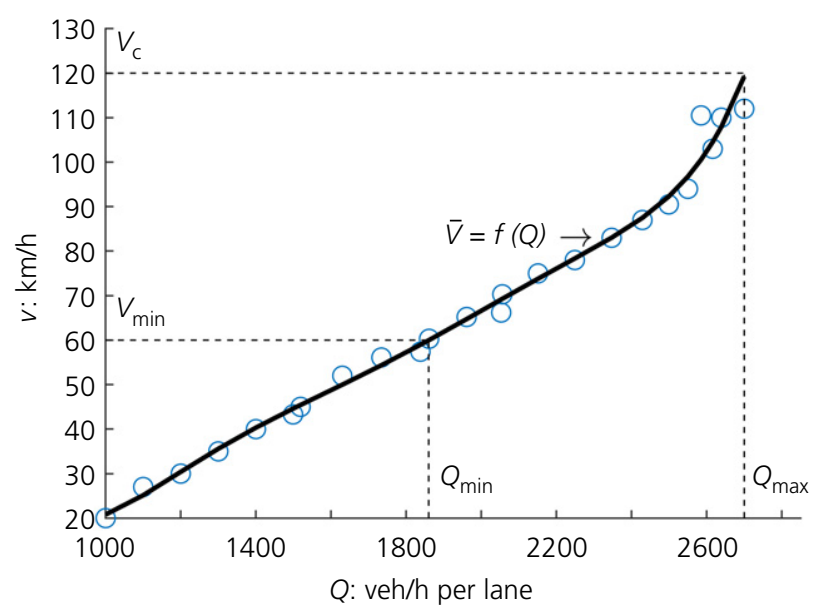

Figure 2. Strictly increasing function $f(Q)$ from $\left(Q_{\min }, Q_{\max }\right)$ to $\left(V_{\min }, V_{c}\right)$

can be mapped into VSLC. This is done by a generalisation of the ALINEA algorithm for ramp metering (Papageorgiou et al., 1991) into a simple control law for VSLC. ALINEA is a local ramp metering algorithm that uses a pure integral control action represented as (Papageorgiou et al., 2008; Smaragdis and Papageorgiou, 2003)

$$
\text { 1. } R\left(k T_{1}\right)=R\left((k-1) T_{1}\right)+K_{\mathrm{r}}\left[O_{\mathrm{d}}-O\left(k T_{1}\right)\right]
$$

where $k$ is the time step, $T_{1}$ is the discretisation time, $R\left(k T_{1}\right)$ is the computed on-ramp flow for the current and $R\left((k-1) T_{1}\right)$ in the previous time step, $K_{\mathrm{r}}$ is a control parameter, $O\left(k T_{1}\right)$ is the measured downstream occupancy at the current time step and $O_{\mathrm{d}}$ is the desired downstream occupancy. $O_{\mathrm{d}}$ is typically chosen close to be the critical occupancy $O_{\mathrm{c}}$.

ALINEA can be generalised into a simple VSLC law if the preceding motorway section is considered as an on-ramp for the next section. The core idea is to map the traffic flow control actions of the preceding motorway section into speed control actions based on the fundamental speed-flow relationship (Figure 2). In this case, the ALINEA algorithm regulates the metered flow rate $Q_{i}$ from section $i-1$ to section $i$ of the urban motorway. The control law is given by (Hadjipollas et al., 2008; Wang, 2011)

$$
\text { 2. } \bar{Q}_{i}\left(k T_{1}\right)=Q_{i}\left((k-1) T_{1}\right)+K_{\mathrm{V}}\left[\rho_{\mathrm{d}}-\frac{1}{N_{\mathrm{r}, i}} \sum_{j \in I_{\mathrm{r}, i}} \rho_{j}\left(k T_{1}\right)\right]
$$

where $Q_{i}\left((k-1) T_{1}\right)$ is the flow from the previous time step, $\rho_{\mathrm{d}}$ is the desired density, $I_{\mathrm{r}, i}$ is the set of downstream sections relevant to section $i, N_{\mathrm{r}, i}$ is the number of sections in the set $I_{\mathrm{r}, i}, \quad K_{\mathrm{v}}$ is the controller gain parameter and $\rho_{j}\left(k T_{1}\right)$ is the density of relevant downstream motorway sections in time step $k$ regarding section $i$. The controller generates its 
command outputs every $T_{1}$ seconds, where $T_{1}=N_{\mathrm{C}} T_{0} . N_{\mathrm{C}}$ is a positive integer (in this paper $N_{\mathrm{C}}=10$ ), and $T_{0}$ is the simulation sampling time (in this paper $T_{0}=30 \mathrm{~s}$ ). The final desired flow rate $Q_{i}$ is determined as

3. $Q_{i}\left(k T_{1}\right)=\left\{\begin{array}{cc}Q_{\max } & \text { if } \bar{Q}_{i}\left(k T_{1}\right) \geq Q_{\max } \\ Q_{\min } & \text { if } \bar{Q}_{i}\left(k T_{1}\right) \leq Q_{\min } \\ \bar{Q}_{i}\left(k T_{1}\right) & \text { otherwise }\end{array}\right.$

where $Q_{\max }$ represents the maximum traffic flow achieved around speed $V_{\mathrm{c}}$, which corresponds to the critical density $\rho_{\mathrm{c}}$, while $Q_{\min }$ is set as the flow corresponding to $V_{\min }$. It is assumed that the inequality $V_{\min } \leq V_{\mathrm{c}} \leq V_{\max }$ is fulfilled ( $V_{\min }$ and $V_{\max }$ are the minimum and maximum allowed speed limits on a particular motorway section).

The speed limit controller has to make decisions based on activities in downstream sections and calculate the new speed limit only if necessary (Hadjipollas et al., 2008; Wang, 2011). This can be implemented by using an appropriate control variable. Let $I_{\mathrm{v}}$ represent the set of road sections in which speed limits are controlled and $C_{i}$ denote a control variable associated with each motorway section $\left(i \in I_{\mathrm{V}}\right)$. The controller generates the desired speed limit $\bar{V}_{i}$ for section $i$ using density
As already mentioned, for mapping purposes, $Q_{\min }$ is set as the flow corresponding to $V_{\min }$ and $Q_{\max }$ is the flow corresponding to the critical density (capacity flow) - that is, $V_{\mathrm{c}}$. Capacity flow is usually not achieved at the maximum allowed speed (Grabec, 2014; Hadjipollas et al., 2008; Papageorgiou et al., 1991) because at higher speeds it is necessary for vehicles to maintain a larger space and time distance (headway) for safety reasons. This reduces the vehicle flow at the observed cross-section of the motorway. The estimated flow-density and speed-density relationships used in this paper are presented in more detail in Section 5. They are used to obtain a speed-flow function that determines the new value of the speed limit (Figure 2)

\section{5. $\quad \bar{V}_{i}\left(k T_{i}\right)=f\left(Q_{i}\left(k T_{i}\right)\right)$}

The new speed limit obtained using Equation 5 can contain significant fluctuations in computed speed limits regarding consecutive control time steps. Such fluctuations can reduce the potential of VSLC for overall speed homogenisation and demotivate drivers to obey the posted speed limits. The following rules are used to compute the final posted speed limit $V_{i}$ to avoid these problems.

$$
\text { 6. } V_{i}\left(k T_{1}\right)= \begin{cases}V_{i}\left((k-1) T_{1}\right)-C_{\mathrm{v}} & \text { if } \bar{V}_{i}\left(k T_{1}\right) \leq V_{i}\left((k-1) T_{1}\right)-C_{\mathrm{v}} \\ V_{i+1}\left(k T_{1}\right)+C_{\mathrm{v}} & \text { if } \bar{V}_{i}\left(k T_{1}\right) \geq V_{i+1}\left(k T_{1}\right)+C_{\mathrm{v}} \\ \bar{V}_{i}\left(k T_{1}\right) & \text { otherwise }\end{cases}
$$

data from the congested section $i+1$ only if the control variable is active (Hadjipollas et al., 2008). To determine whether the control variable $C_{i}$ is active or not, the density in the downstream section $\left(\rho_{i+1}\right)$ at a particular moment $\left(k T_{1}\right)$ has to be measured. The following three cases have to be observed for this decision.

- If $\rho_{i+1}\left(k T_{1}\right) \geq\left(1+\Delta_{+}\right) \rho_{\mathrm{c}}$ then $C_{i}$ is active $\left(\Delta_{+}\right.$is a activation design parameter and $\rho_{\mathrm{c}}$ is the critical density).

- If $\rho_{i+1}\left(k T_{1}\right) \leq\left(1-\Delta_{-}\right) \rho_{\mathrm{c}}$ then $C_{i}$ is inactive $\left(\Delta_{-}\right.$is a deactivation design parameter).

- If neither of these two inequalities is satisfied, $C_{i}$ maintains its status as in the previous time step.

Flow commands, computed using Equations 2 and 3, must be mapped into speed limit commands to control the traffic speed instead of traffic flow. This is done by using the flow-speed relationship shown in Figure 2. The speed limit of the traffic flow in section $i$ has to be bounded due to traffic regulations as (Kušić et al., 2016)

4. $V_{\min } \leq V_{i}(k T) \leq V_{\max }$ where $C_{\mathrm{v}}$ is a positive constant that represents the maximum allowed change in speed limit between two consecutive control time steps (usually $10 \mathrm{~km} / \mathrm{h}$, as also used in this paper) and $V_{i}\left(k T_{1}\right)$ is the final speed limit for motorway section $i$ (Carlson et al., 2010).

\subsection{Simple proportional speed controller (SPSC)}

The MVM controller changes speed limits in situations when the chosen critical density is exceeded. A drawback of this approach is that the value of the desired density $\left(\rho_{\mathrm{d}}\right)$ can be set much lower than the critical density and the controller's response may be too strong and vice versa. A second drawback is related to the need to estimate the fundamental diagram, which must be measured at the particular controlled motorway segment. It can take a lot of work to estimate traffic characteristics if the various geometry characteristics have to be included (number of lanes, entrances and curvature of the motorway segment) since they impact traffic flow. Moreover, a change in the speed limit on motorways is only necessary when a significant disturbance occurs (Hadjipollas et al., 2008). Frequent reductions of posted speed limits in situations when significant disturbances are not present can have an adverse effect on the overall motorway traffic system. This is why it is necessary to 
create a VSLC controller that can adequately respond to changes in downstream density instead of maintaining a fixed desired density. The SPSC contains this characteristic and is a simplified version of the MVM approach (Wang, 2011).

Let us suppose that a disturbance has occurred in the most downstream section of the controlled motorway segment. The speed controller implemented upstream (section $i$ ) should be active and react to downstream density changes (Carlson et al., 2010). The SPSC uses the same control variables for activation as the MVM controller. If the control variable $C_{i}$ is in an active state, computation of the new speed limit is required, given by

\subsection{Vissim and Enviver simulators}

The traffic simulation tool Vissim is a microscopic simulation environment. In microscopic simulations, each entity (car, truck, train, pedestrian etc.) is simulated on an individual level. Vissim uses for this the Wiedmann model (Higgs et al., 2011) based on the interaction between entities depending on the psychophysical reactions of the driver. Each of the entities in the simulation is represented by a separate entity with all of its associated attributes. The latter describes the behaviour characteristics of a single vehicle pair in the simulated traffic flow, with the assumption that such behaviour can be applied to every other pair of vehicles. Thus, separate vehicle characteristics are used that can

7. $V_{i}\left(k T_{1}\right)= \begin{cases}V_{i}\left((k-1) T_{1}\right)-C_{\mathrm{v}} & \text { if } \bar{V}_{i}\left(k T_{1}\right) \leq V_{i}\left((k-1) T_{1}\right)-C_{\mathrm{v}} \\ V_{i}\left(k T_{1}\right)+C_{\mathrm{v}} & \text { if } \bar{V}_{i}\left(k T_{1}\right) \geq V_{i}\left(k T_{1}\right)+C_{\mathrm{v}} \\ \bar{V}_{i}\left(k T_{1}\right) & \text { otherwise }\end{cases}$

The speed limit in each motorway section is again bounded using Equation 4. In Equation 8 the difference between the density from the previous time step $(k-1)$ and the current density of all affected downstream sections with respect to section $i$ is added to the speed limit $V_{i}\left((k-1) T_{1}\right)$ from the previous time step (Wang, 2011). The obtained result is the new speed limit $\bar{V}_{i}(k T)$

$$
\text { 8. } \begin{aligned}
\quad \bar{V}_{i}\left(k T_{1}\right)= & V_{i}\left((k-1) T_{1}\right) \\
& +K_{\mathrm{v}}\left[\sum_{i=1}^{N-1} \rho_{i+1}\left((k-1) T_{1}\right)-\sum_{i=1}^{N-1} \rho_{i+1}\left(k T_{1}\right)\right]
\end{aligned}
$$

\section{Simulation framework}

Both controllers are analysed using the microscopic traffic simulator Vissim, vehicle emission simulator Enviver and the programming platform Matlab for implementation of the speed limit controllers. The framework is also used to compare the effects of changing the control gain parameter $K_{\mathrm{v}}$. The block scheme of the implemented simulation framework consisting of the mentioned software packages is given in Figure 3.

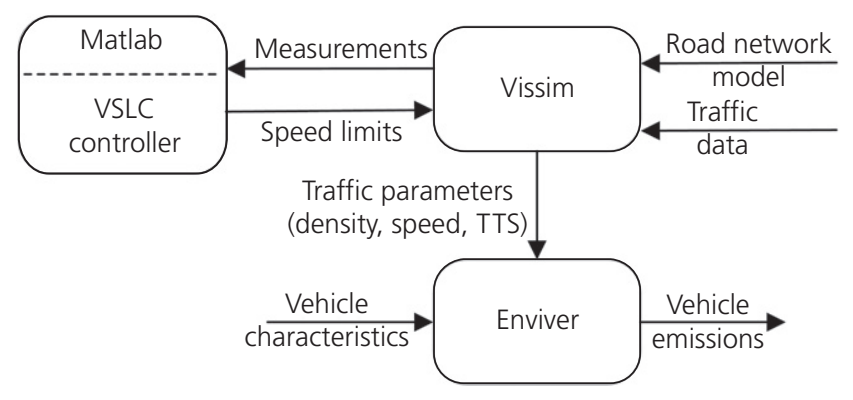

Figure 3. Block scheme of the simulation framework (Kušić et al., 2016) describe the motion of different vehicle types (e.g. speed, headway and acceleration) (PTV Group, 2019).

Enviver is a software based on the Versit + exhaust gas emissions model (TNO, 2019) that enables researchers to study the exhaust emissions of traffic scenarios simulated in Vissim. For this, after each Vissim simulation, the collected data (speeds, accelerations, number of vehicles, vehicle types, a simulation second, vehicle position etc.) are loaded into Enviver. Based on these imported data, Enviver computes vehicle emissions for the simulated traffic scenario. Thus, there is no real-time connection between the control process and Enviver. It is only used for the evaluation of the VSLC impact on vehicle emissions after the simulation has finished. Enviver can calculate the emission rates of several different exhaust gases (carbon dioxide $\left(\mathrm{CO}_{2}\right)$ and nitrogen oxides $\left(\mathrm{NO}_{x}\right)$ ) and particulate matter (TNO, 2019).

\subsection{Connecting Vissim, Matlab and Enviver}

The microscopic simulators Vissim and Matlab were connected through the Com interface, enabling them to exchange data in real time using the framework shown in Figure 3. Matlab manages the simulation. Traffic flow parameters (speed, density and flow) are collected and calculated using appropriate functions created in Matlab, which has access to detectors in Vissim simulating the work of a real traffic controller. The current speed limit decisions proposed by the VSLC controllers mentioned earlier are sent directly to the corresponding VMSs in Vissim. Vissim records all the required data for calculation of exhaust emissions into an output file after the simulation ends. These data are then imported into the software Enviver to obtain vehicle emissions from traffic observed during the simulation (TNO, 2019).

\subsection{Measuring average speed, flow and density}

Since the fundamental diagram is based on the flow-density relationship, these two quantities need to be measured. The 
first step is to define the geometrical characteristics of the simulated motorway model. These include the location of onand off-ramps, the number of lanes, the locations of sensors and so on. The simulated motorway segment is divided into smaller segments, usually called sections or cells. The next step is data collection. Real-time traffic data are collected directly from sensors placed within the motorway model created in Vissim. Each sensor sends impulses when a vehicle is present. It can also measure the current speed of a vehicle passing the sensor. Based on these two 'impulse and speed' measurements, it is possible to compute all the necessary traffic parameters for a motorway section such as density, flow and average speed.

The mentioned traffic data are collected consecutively during each sampling time interval. The required traffic parameters (density, speed and flow) are obtained using Equations 9-11. From data collected from a larger number of simulations, it is possible to estimate additional information such as the freeflow speed, capacity and critical and jam densities, which are the input parameters for the two analysed controllers. The traffic density at a particular section of the motorway can be obtained using

9. $\rho_{i}\left(k T_{0}\right)=\rho_{i}\left((k-1) T_{0}\right)+\left(\frac{n_{\mathrm{in}_{i}}\left(k T_{0}\right)-n_{\mathrm{out}_{i}}\left(k T_{0}\right)}{l_{i}}\right)$

where $\rho_{i}\left(k T_{0}\right)$ is the current density in section $i$ at time step $k$ and $\rho_{i}\left((k-1) T_{0}\right)$ is the density from the previous time step $k-1$. The term in parentheses represents the difference between the number of incoming vehicles $\left(n_{\mathrm{in}_{i}}\left(k T_{0}\right)\right)$ and outgoing vehicles $\left(n_{\text {out }_{i}}\left(k T_{0}\right)\right)$, regarding section $i$. It is divided by the section length $l_{i}$. The speed is calculated as the arithmetic mean speed measured at the beginning and the end of the particular section $i$

10. $\quad \bar{v}_{i}\left(k T_{0}\right)=\frac{\bar{v}_{\text {in }}\left(k T_{0}\right)+\bar{v}_{\text {out }}\left(k T_{0}\right)}{2}$

where $\bar{v}_{i}\left(k T_{0}\right)$ is the average speed at section $i$ in time step $k T_{0}$, $\bar{v}_{\text {in }}\left(k T_{0}\right)$ is the average speed at the beginning of section $i$ and $\bar{v}_{\text {out }}\left(k T_{0}\right)$ is the average speed at the end of section $I$, both measured between time steps $(k-1) T_{0}$ and $k T_{0}$. The traffic flow in motorway section $i$ at time $k T_{0}$ is

11. $Q_{i}\left(k T_{0}\right)=\frac{n_{i}}{T_{0} / 3600}$

where $Q_{i}\left(k T_{0}\right)$ is the traffic flow across section $i$ in the time interval converted into units of vehicles per hour (veh/h) and $n_{i}$ is the number of vehicles that passed through the crosssection at the end of motorway section $i$ measured during time steps $(k-1) T_{0}$ and $k T_{0}$.

\section{Measurement of the fundamental diagram}

As already mentioned, the MVM controller uses the fundamental diagram to map traffic flow values into speed limits. To do this, the fundamental diagram of the controlled motorway segment has to be constructed. The method applied for measurement of the fundamental diagram requires several steps. First, traffic data (velocity, flow and density) are generated and collected within the simulator Vissim. These measurements are used to determine the boundary that separates the collected traffic data into two states. The first state represents the free-flow condition and the second state represents the congested traffic condition. At the separation line between these two states, values of $Q_{\max }$ and $V_{\mathrm{c}}$ can be measured. The procedure itself is described in more detail elsewhere (Dervisoglu et al., 2009). It should be noted that the calibration procedure of the simulated segment of motorway is not included here because a synthetic model with corresponding traffic flow was used in this work. The purpose of this research is to establish a methodology for tuning a controller for VSLC and to evaluate the impacts on traffic flow and vehicle emissions.

The free-flow speed is estimated by performing a least-squares fit on the flow-density data in the range where the free-flow speed is reported to be above $105 \mathrm{~km} / \mathrm{h}$. A scatterplot of the collected flow-density pairs used to obtain the fundamental diagram with the amount of collected data is shown in Figure 4. The maximum value of flow across the motorway section from all observed measurements is assigned as the capacity of that section. This value is horizontally projected according to the free-flow line to establish the tip of the triangular fundamental diagram. The obtained intersection represents the critical density for the motorway section. After that point, traffic flow becomes unstable and the probability of congestion increases. Other non-linear approaches can also be used for estimation of the characteristics of the fundamental

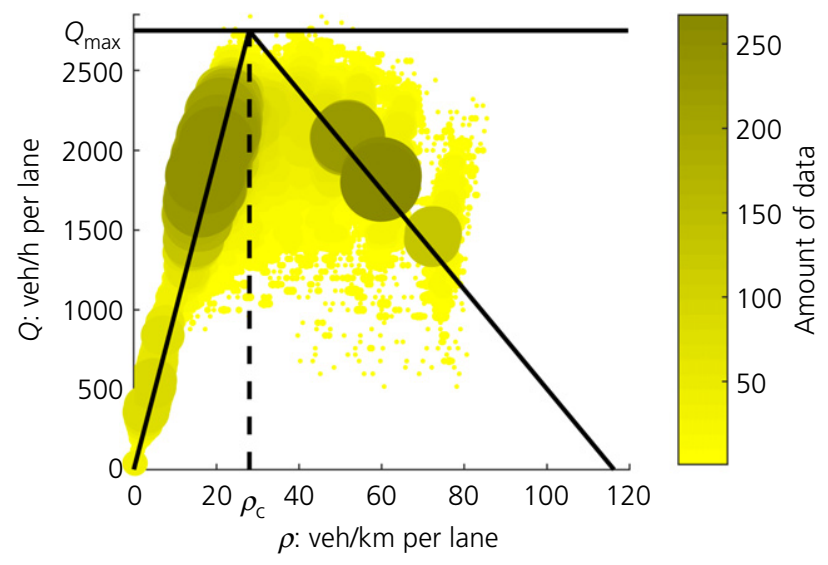

Figure 4. Obtained triangular fundamental diagram 


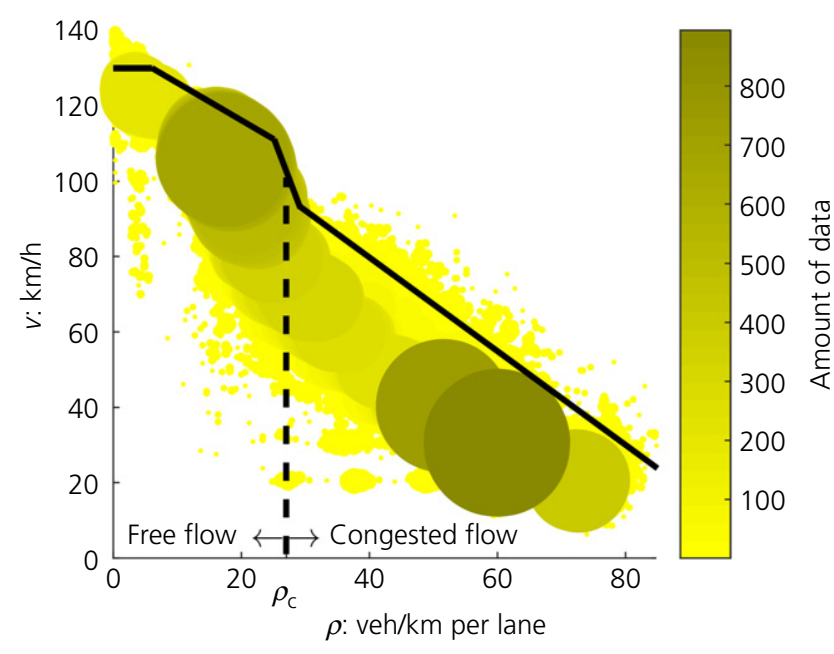

Figure 5. Obtained speed-density relationship

diagram. One such approach, based on polynomial approximation, is presented by Rosas-Jaimes et al. (2016).

The scale bar on the right-hand side of Figure 4 shows the number of measurements collected during multiple simulations for a particular flow-density pair. Larger amounts of data are represented by the darker shades and smaller amounts of data by lighter shades. To the left of the critical density is the area of free-flow traffic; to the right is the area of congested traffic. Flow-density data pairs on the line falling on the right-hand side of the triangular fundamental diagram are used to obtain the corresponding speed for congested traffic flow (Figure 5). The piecewise affine function (black line) drawn through these data represents the speed-density dependence. The interesting part is the congested side of the diagram $\left(\rho \geq \rho_{\mathrm{c}}\right)$ whose values are used to correlate the data from $Q\left(\rho \geq \rho_{\mathrm{c}}\right)$ and $v\left(\rho \geq \rho_{\mathrm{c}}\right)$ into the required mapping function denoted with Equation 5. A polynomial function of sixth order was used for approximation and its coefficients were obtained using the leastsquares method. The resulting function is shown in Figure 2, where the circles denote the speed-flow pairs used for the function approximation. It should be noted that only density can be applied for accurate recognition of congested traffic flow a small traffic flow can denote both scarce traffic or severe congestion.

\section{Results}

This section presents the results of a comparison of the chosen VSLC controllers with the case of no control regarding the impact on both traffic flow and the environment. For an in-depth analysis, changes in the controller gain parameter $K_{\mathrm{v}}$ were applied. The step size was chosen to be $0 \cdot 5$ in the interval $0 \leq K_{\mathrm{v}} \leq 10$. In all the simulations, the parameters of the analysed controllers were set to the following values: $V_{\text {min }}=60 \mathrm{~km} / \mathrm{h}, \quad V_{\max }=130 \mathrm{~km} / \mathrm{h}, \quad Q_{\min }=1850 \mathrm{veh} / \mathrm{h}$ per lane, $Q_{\max }=2700 \mathrm{veh} / \mathrm{h}$ per lane, $\Delta_{+}=-0.055, \Delta_{-}=0 \cdot 342$, $\rho_{\mathrm{c}}=\rho_{\mathrm{d}}=28 \mathrm{veh} / \mathrm{km}$ per lane, $K_{\mathrm{v}} \in[0,10], C_{\mathrm{v}}=10 \mathrm{~km} / \mathrm{h}$ and $T_{1}=5 \mathrm{~min}$.

To measure the LoS, appropriate MoE have to be chosen. In this work, TT and TTS were used for traffic-related LoS. TT measures how much time one vehicle needs to travel through an observed motorway segment and is related to mainstream traffic only. TTS represents the amount of time spent by all of the vehicles on the motorway segment, including waiting times on all ramps. For the environmental part, vehicle emissions regarding carbon dioxide, nitrogen oxides and particulate matter with a mean diameter of $10 \mu \mathrm{m}\left(\mathrm{PM}_{10}\right)$ were used, as in previous work (Kušić et al., 2016). These selected MoE can describe the situation on an urban motorway in more detail than the usually used density LoS measure from the Highway Capacity Manual (TRB, 2000). This detailed description is crucial for the analysis in this paper. Also, it should be noted that, for a real traffic scenario, the simulated model has to be calibrated based on real traffic demand data. Accordingly, the improvements for a real motorway system could differ from the improvements obtained in this analysis.

\subsection{Motorway model and traffic data}

A synthetic model of a three-lane urban motorway (Figure 6) was constructed in the microscopic simulator Vissim. The model was divided into four sections. VSLC was applied in sections $L_{1}(1 \mathrm{~km}), L_{2}(0.8 \mathrm{~km})$ and $L_{3}(0.7 \mathrm{~km})$; the last section $L_{4}(2 \mathrm{~km})$ was without VSLC. Section $L_{2}$ contains one on-ramp and one off-ramp $\left(\mathrm{r}_{1}\right.$ and $\left.\mathrm{s}_{1}\right)$ while section $L_{4}$ has only one on-ramp $\left(r_{2}\right)$. The model and input traffic flow data were based on the model of Kušić et al. (2016) with small changes to enable better analysis. The changes made relate to the lengths of sections $L_{2}$ and $L_{3}$, so ramps $\mathrm{r}_{1}$ and $\mathrm{s}_{1}$ are now located in section $L_{2}$. In addition, compared with the original model of Kušić et al. (2016), the mainstream and traffic demand at the first on-ramp were, on average, increased by $10 \%$.

VMSs were located at the beginning of sections $L_{1}, L_{2}$ and $L_{3}$; these are denoted as vertical rectangles in Figure 6. The VMSs were used to inform drivers about the currently allowed speed limit in a particular section. The traffic flow was set to consist of $96 \%$ cars, $2 \%$ trucks and $2 \%$ buses. The main traffic flow had a constant rate of $4500 \mathrm{veh} / \mathrm{h}$ during the whole simulation, out of which $95 \%$ of traffic remained on the main road while the other $5 \%$ left the mainstream through off-ramp $s_{1}$. On-ramp $r_{1}$ had a constant traffic demand of $1350 \mathrm{veh} / \mathrm{h}$, while the traffic demand on on-ramp $r_{2}$ was changed during simulation, as shown in Figure 7. As already mentioned, the input traffic data used were synthetic and the calibration process of the simulated model is not included in this paper. The simulation parameters (acceleration, time headway etc.) in the simulated model were set to reflect traffic on motorways according to Leyn and Vortisch (2015). 


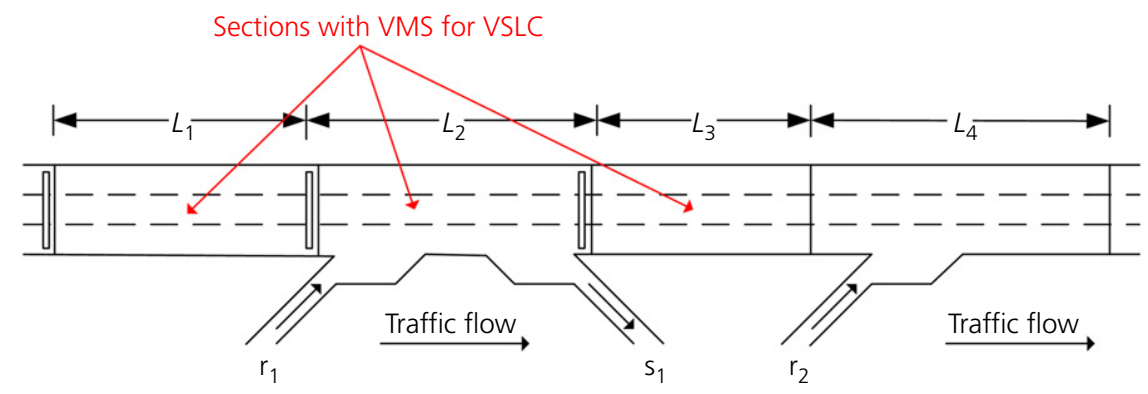

Figure 6. Unidirectional motorway segment divided into four sections (Kušić et al., 2016)

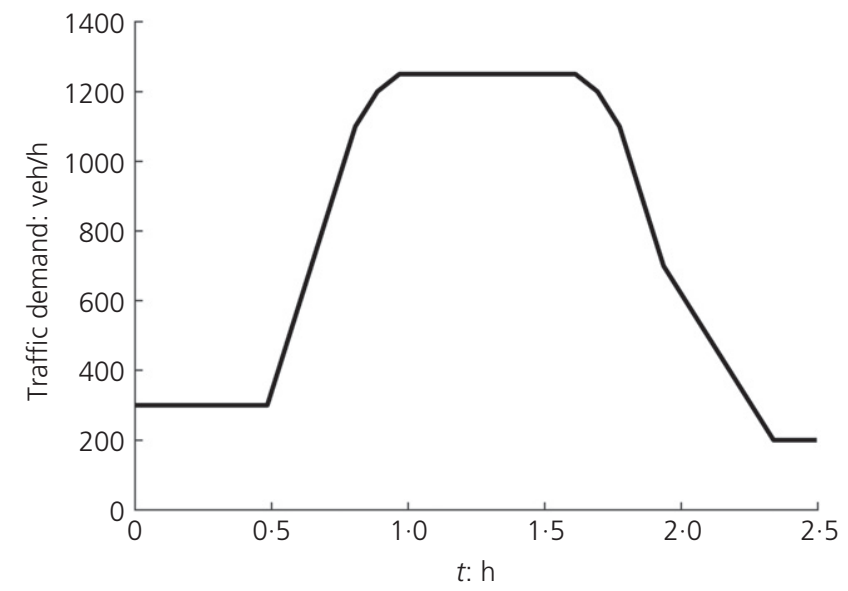

Figure 7. Traffic demand on on-ramp $r_{2}$ during the simulation

The maximum traffic load on on-ramp $r_{2}$ of $1250 \mathrm{veh} / \mathrm{h}$ was achieved around the first hour of the simulation and lasted for about $30 \mathrm{~min}$. With this traffic demand, traffic congestion was created in the area near on-ramp $r_{2}$. This congestion gradually propagated upstream and produced a traffic disturbance in the mainstream flow. The traffic congestion created was the primary test for the VSLC controllers analysed.

\subsection{Traffic parameters}

Figures 8 and 9 show the influence of parameter $K_{\mathrm{v}}$ on TTS and TT for the case with no VSLC, the MVM controller and the SPSC. The application of VSLC reduced TTS and TT by about $5 \%$ depending on the value of the parameter $K_{\mathrm{v}}$. Small values of $K_{\mathrm{v}}$ were found to be favourable for the MVM controller and values from 4 to $4 \cdot 5$ were favourable for the SPSC. Large values of $K_{\mathrm{V}}$ did not improve TTS and TT since both controllers significantly reduced the speed limit very quickly to the lowest allowed value $\left(V_{\min }=60 \mathrm{~km} / \mathrm{h}\right)$, resulting in a negative influence on the LoS compared with the case with no VSLC.

Figure 10 shows the typical traffic flow $(q)$, density $(\rho)$ and average speed ( $v$ ) measured in sections $L_{2}, L_{3}$ and $L_{4}$ during the simulation. The most significant changes and the impact of VSLC can be seen. Section $L_{1}$ is not shown in the figure as

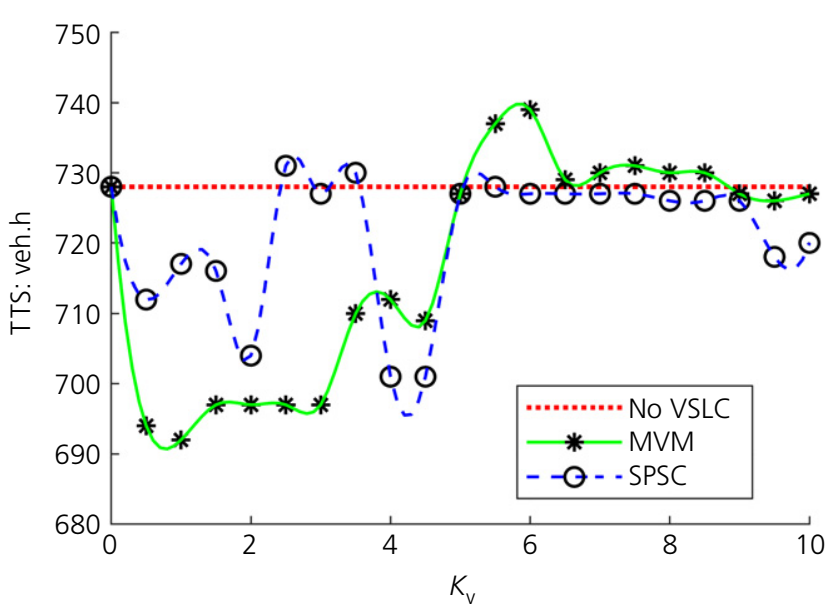

Figure 8. Influence of controller gain parameter $K_{\mathrm{v}}$ on TTS

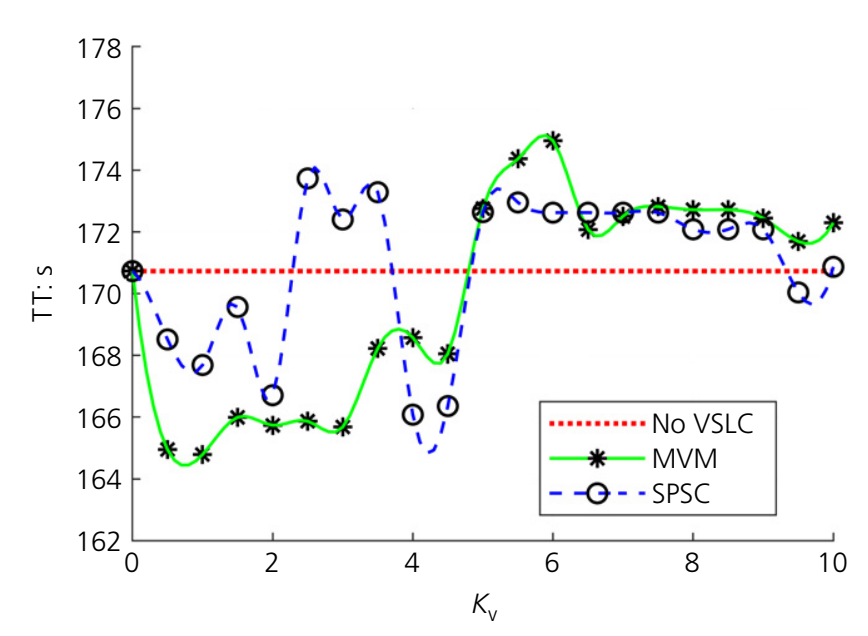

Figure 9. Influence of controller gain parameter $K_{\mathrm{v}}$ on TT

negligible changes in the traffic parameters were found when VSLC was applied compared with the case without.

In the density plots for sections $L_{3}$ and $L_{4}$, the effect of VSLC is evident, with actions that actively kept the density within acceptable values. For the simulated motorway segment, the critical density $\rho_{\mathrm{c}}$ was $28 \mathrm{veh} / \mathrm{km}$ per lane, as shown by the 

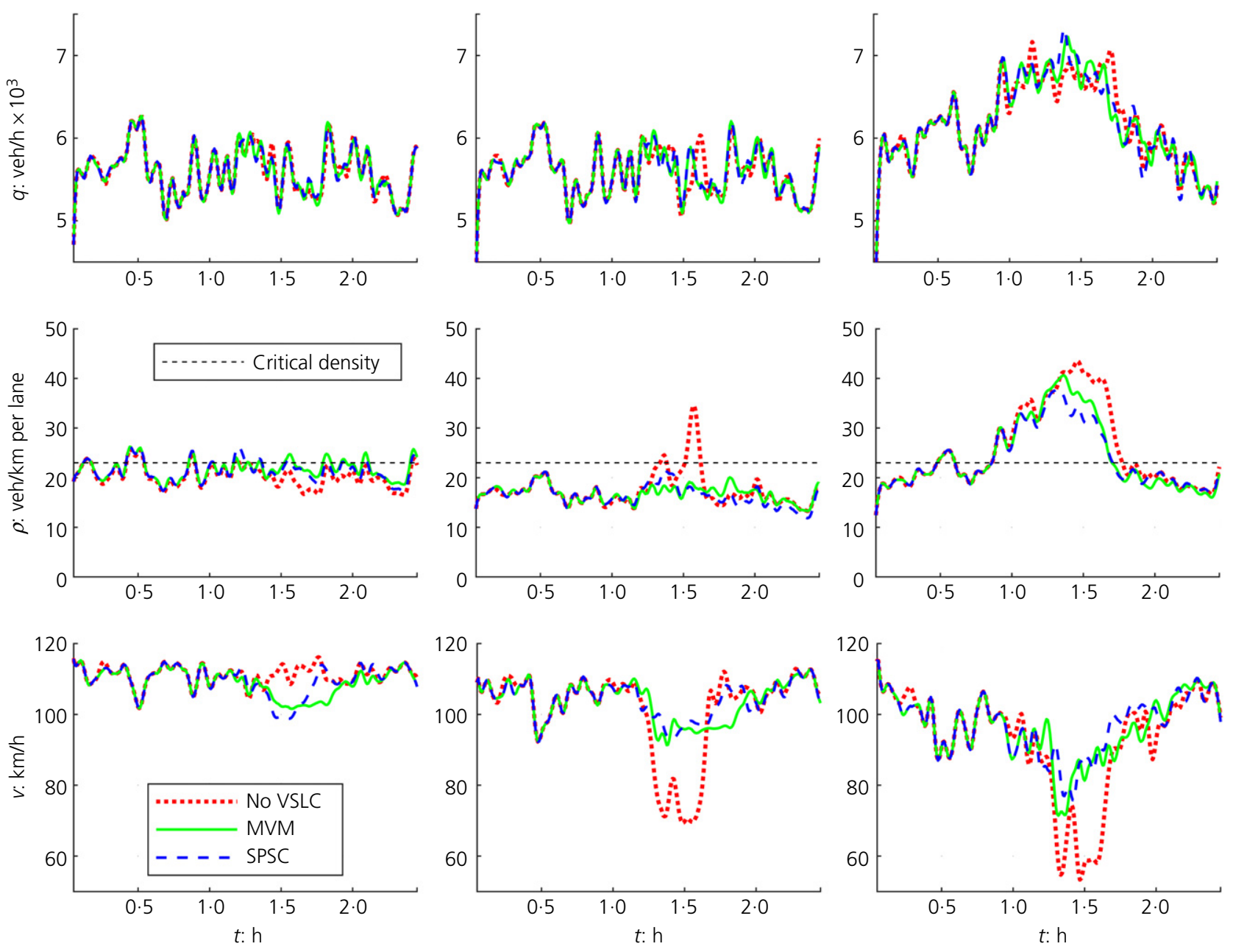

Figure 10. Obtained flow (top), density (middle) and average speed (bottom) in sections $L_{2}$ (left), $L_{3}$ (middle) and $L_{4}$ (right)

dashed lines in the middle row of plots in Figure 10. By reducing the density of traffic, higher average speeds were achieved in sections $L_{3}$ and $L_{4}$. VSLC managed to keep the density of traffic flow in section $L_{3}$ under the critical value during the whole simulation. Without VSLC, the density in section $L_{4}$ significantly exceeded the critical density $\rho_{\mathrm{c}}$. Both controllers were able to reduce that.

By reducing such large changes in traffic density, the formation of major congestion can be prevented. This consequently reduces the sudden deceleration and acceleration of vehicles, resulting in reduced fuel consumption and lower exhaust emissions because of the more homogeneous traffic speed (Carlson et al., 2010). In the case of very high traffic demand, the controllers could not prevent congestion build-up (as can be seen in section $L_{4}$ ), which indicates the limitations of VSLC during very high traffic demand. Nevertheless, both controllers still reduced the increase in density compared with the case of no VSLC and ensured higher traffic speeds.
The traffic flows showed similar behaviour in all three cases. Only in section $L_{4}$ was an increase in traffic flow observed in all the simulated cases. This was during the period with high traffic demand as a consequence of the lower density and higher speed.

Both the MVM controller and the SPSC induced lower speed limits for the mainstream flow during the time of higher onramp demand, all with the aim of keeping the density within acceptable values if possible. This is evident in Figures 11 and 12, which show the output values of the VSLC controllers. First, the controller in section $L_{3}$ started to reduce the speed limit since this controller was closest to the congestion in section $L_{4}$. Its reaction corresponds to the increased traffic demand resulting from the traffic flow entering the mainstream at on-ramp $r_{2}$. The controllers in the two previous sections $\left(L_{1}\right.$ and $L_{2}$ ) followed, with a reduction in the speed limit and postponing the arrival of upstream vehicles to the congested area. Their speed limit reductions were lower as they were further 


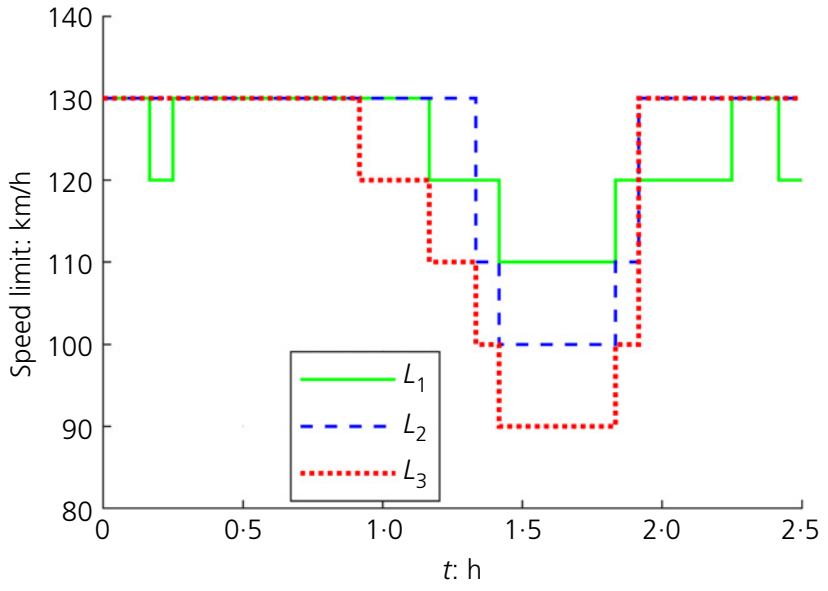

Figure 11. Speed limit change with the MVM controller

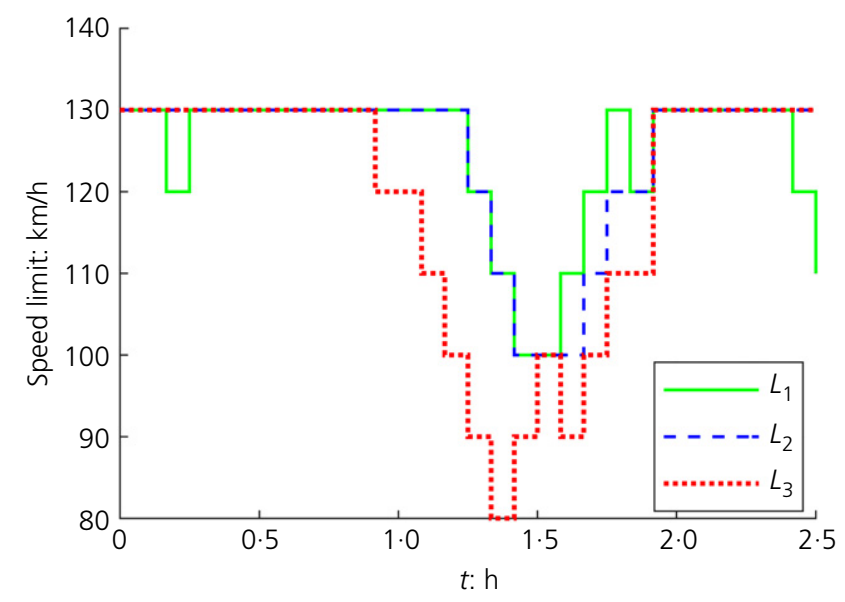

Figure 12. Speed limit change with the SPSC

away from the congested area. The MVM controller reduced the speed limit to lower values than the SPSC and the speed limit in the previous sections was also reduced earlier. Consequently, the MVM controller had, on average, lower TTS and TT values (Figures 8 and 9), especially for low values of the controller gain parameter $K_{\mathrm{v}}$.

VSLC also impacted the queue lengths on the on-ramps. This effect is shown for on-ramp $r_{2}$ for a typical simulation in Figure 13. Due to the congestion in section $L_{4}$, the queue length increased. Without VSLC there were larger oscillations in the queue length and the longer queues lasted for a longer period of time. Since VSLC reduces the mainstream traffic density and creates a more homogeneous speed of the traffic flow, vehicles from on-ramps can merge more easily into the mainstream traffic, resulting in a shorter queue length. This explains the shorter queue length for the SPSC since it produced a lower traffic density in section $L_{4}$, as can be seen in Figures 10 and 13 .

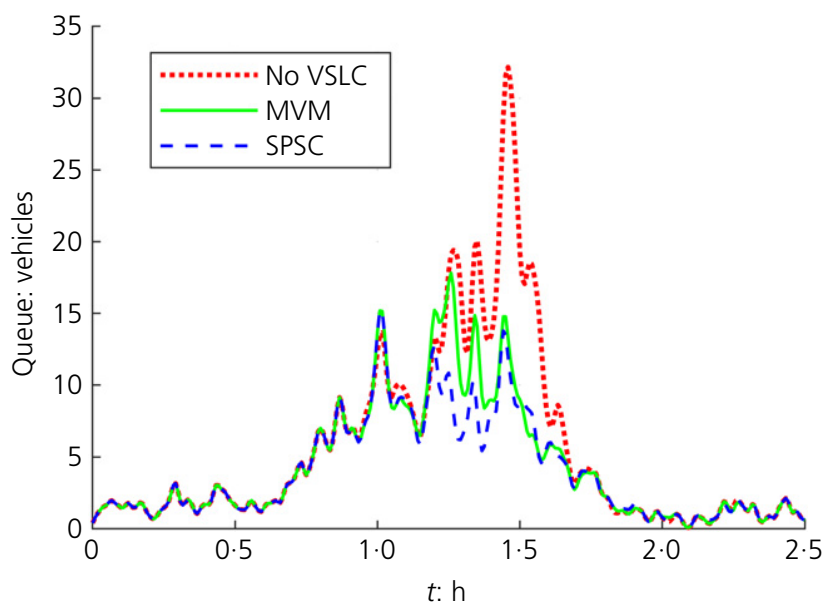

Figure 13. Queue length at on-ramp $r_{2}$

\subsection{Vehicle emissions}

The vehicle emissions analysed in this work were carbon dioxide and nitrogen oxides; the harmful particles analysed were $\mathrm{PM}_{10}$. A reduction in vehicle emissions in this analysis is not a part of the control process but a consequence. The chosen controllers only aim to improve the traffic situation. External software Enviver was used only for analysis of the final output data obtained after the simulations in order to show the influence of VSLC on vehicle emissions. Emissions depend on the traffic condition, which is dynamically influenced by VSLC. The characteristics of exhaust emissions related to vehicle speed are reported by Brandt et al. (2012).

The influence of the parameter $K_{\mathrm{v}}$ on carbon dioxide, nitrogen oxides and $\mathrm{PM}_{10}$ is shown in Figures 14-16. The results show that the application of VSLC reduced vehicle emissions for all values of $K_{\mathrm{v}}$ but reduced $\mathrm{PM}_{10}$ only for lower $K_{\mathrm{v}}$ values. A reduction in the mainstream speed also reduced the amount of carbon dioxide emissions for both controllers. For $K_{\mathrm{v}}<4 \cdot 5$, carbon dioxide emissions decreased. Beyond this value, the emissions slightly increased due to the higher response of the controllers according to the higher value of $K_{\mathrm{v}}$. Imposed lower speed limits produced longer TTs, which implies more carbon dioxide emissions. As can be seen in Figure 14, the optimal $K_{\mathrm{v}}$ value for both controllers was around $4 \cdot 5$.

In the case of nitrogen oxides (Figure 15), the emissions for both controllers gradually decreased with increasing $K_{\mathrm{v}}$. This phenomenon is related to the characteristics of internal combustion engines, which emit lower emissions of nitrogen oxides at lower vehicle speed. The lower vehicle speed is related to higher values of $K_{\mathrm{v}}$, which produced a stronger response of the controllers (i.e. lower speed limits) during the congested period.

Vehicles also emit particulate matter due to the abrasion processes of the road, tyres and brake wear, depending on engine 


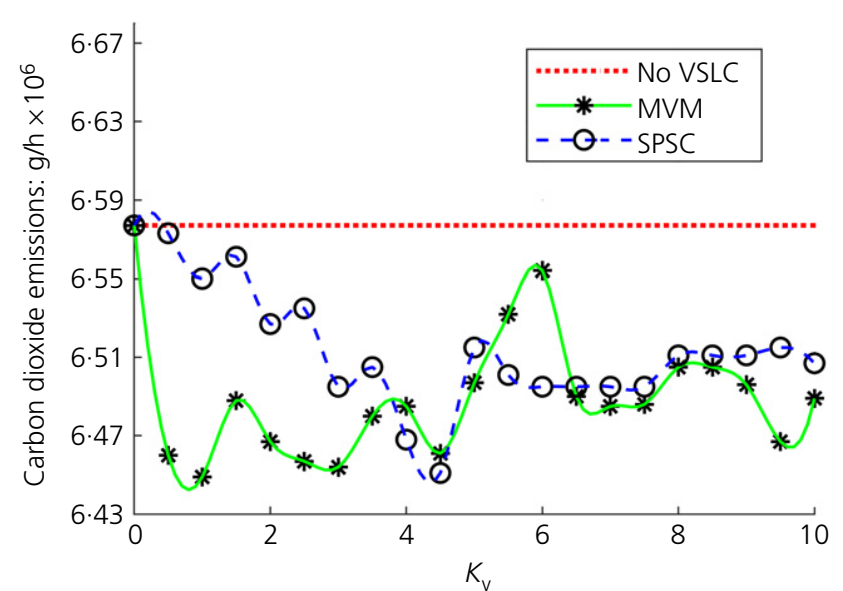

Figure 14. Influence of controller gain parameter $K_{\mathrm{v}}$ on carbon dioxide emissions

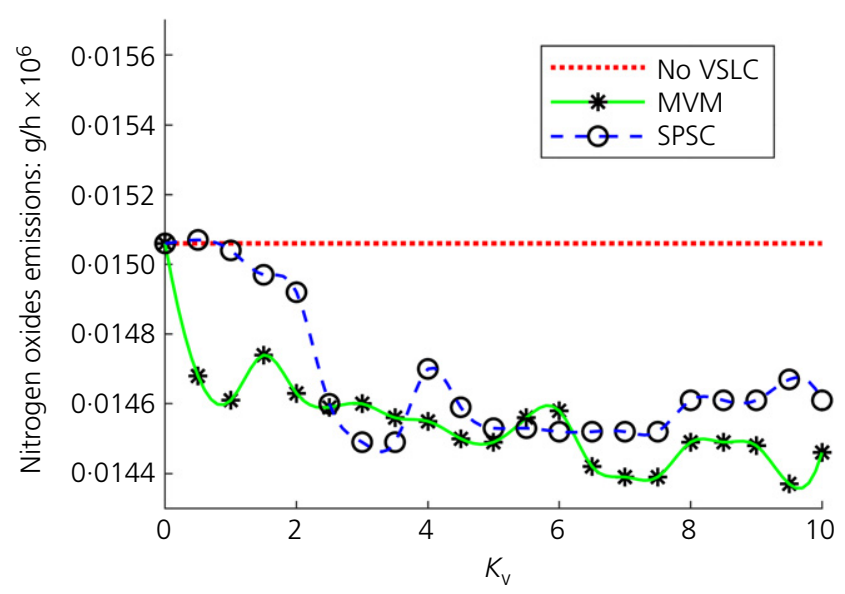

Figure 15. Influence of controller gain parameter $K_{v}$ on nitrogen oxides emissions

revs and load (exhaust) (Brandt et al., 2012). As shown in Figure 16, the equilibrium with the smallest amount of $\mathrm{PM}_{10}$ for SPSC was achieved at around $K_{\mathrm{v}}=4 \cdot 5$. For the MVM controller, a lower value of $K_{\mathrm{v}}$ was found to be preferable. The faster decrease of speed limits according to the controller's larger response due to higher values of the control parameter $\left(K_{\mathrm{v}}>5\right)$ increased the $\mathrm{PM}_{10}$ pollution for both controllers. This is related to the lower allowed speed and, consequently, shorter headways between vehicles, which induces a higher density per unit length of the motorway segments. Such concentrated traffic flow produces higher pollution levels regarding $\mathrm{PM}_{10}$.

\subsection{Discussion}

The working principle of both VSLC controllers is essentially based on the timely detection of congestion (increased density) in traffic flow and then taking preventive action by changing

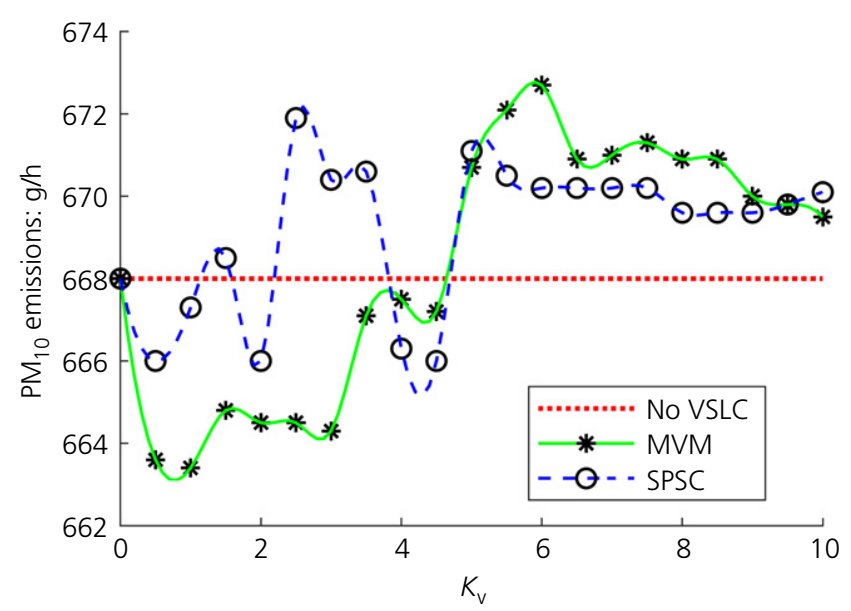

Figure 16. Influence of controller gain parameter $K_{\mathrm{v}}$ on $\mathrm{PM}_{10}$ emissions

the speed limit. The aim is to keep all the traffic parameters within acceptable values. By applying VSLC, the density of traffic flow in section $L_{3}$ during peak traffic demand load on on-ramp $r_{2}$ was maintained within acceptable limits. The critical density was not exceeded in section $L_{3}$ and the situation in section $L_{4}$ was improved. In the case without VSLC, the density in section $L_{3}$ and especially in section $L_{4}$ significantly exceeded the critical density $\rho_{\mathrm{c}}$. The consequence of this is traffic slowdown and increased values of TTS and TT.

VSLC slowed down the vehicles in sections prior to the problematic section, thus allowing more time for the congestion to dissolve. Mainstream vehicles arrived later, giving a chance for vehicles originating from on-ramp $r_{2}$ to merge into the mainstream flow with less negative influence on this flow. With the lower density values in section $L_{4}$ when VSLC was applied, the formation of congestion was reduced in the simulated scenario. This reduced sudden decelerations and accelerations of vehicles, resulting in reduced fuel consumption and lower exhaust emissions (Carlson et al., 2010). The positive influence of the controllers on TTS was up to $5 \%$, on TT up to $3.5 \%$, on carbon dioxide emissions up to $2 \%$, on nitrogen oxides emissions up to $4 \cdot 5 \%$ and on $\mathrm{PM}_{10}$ up to $0 \cdot 7 \%$.

The MVM controller produced better results than the SPSC because the MVM controller has the goal of keeping traffic density near the desired density (usually close to the critical density to ensure maximum throughput). In addition, the MVM controller is active the whole time and reacts earlier with lower limitations in the preceding sections than the SPSC. The SPSC computes the speed limit based on traffic density changes and is only activated when the traffic density is higher than the critical density. Its reaction thus kicks in a little later and therefore demands a larger decrease in the speed limit. The reductions in vehicle emissions confirmed that traffic control can simultaneously fulfil several criteria even when the 
controller used is designed to fulfil only one (improvement of traffic-related parameters). A positive effect on one aspect of traffic flow (TTS and TT) improves the environmental aspect (vehicle emissions) in this case. This is important for ensuring that current road infrastructure can be augmented with systems from the domain of intelligent transportation systems to align it with the EU goals of creating a smart, green and integrated transport environment.

The MVM controller produced a less aggressive and less oscillating speed limit output compared with the SPSC. However, the MVM controller requires construction of a fundamental diagram since it computes the speed limit based on the difference between a fixed desired density $\rho_{\mathrm{d}}$ and the actual downstream density and the flow-speed mapping function. The SPSC responds to changes in downstream density instead of a fixed desired density. In the case of a smaller change in density between the current and previous control time interval, the output speed limit can oscillate, which causes unstable traffic conditions when the SPSC is used. This results in a smaller range of acceptable values of the controller gain parameter $K_{\mathrm{v}}$.

\section{Conclusions}

Two controllers for VSLC were compared regarding different settings of the control gain parameter $K_{\mathrm{v}}$, which influences the effectiveness of the analysed controllers. A description of how to collect and process the required traffic data to obtain the fundamental diagram for the controller set-up procedure was provided. The simple reactive speed limit controllers MVM and SPSC were compared in scenarios with and without VSLC. A comparative analysis was conducted using a synthetic urban motorway segment containing two on-ramps and one off-ramp. The same procedure could be applied on a real urban motorway in order to optimally set the controller gain parameter $K_{\mathrm{v}}$. This is important because traffic flow characteristics depend on driver behaviour and different motorway segments can require different controller settings.

The results showed that both VSLC controllers increased the LoS of the controlled motorway segment in terms of reducing selected MoE related to traffic parameters (TTS and TT) and environmental parameters (vehicle emissions of carbon dioxide, nitrogen oxides and $\mathrm{PM}_{10}$ ) compared with the situation without VSLC applied in an increased traffic demand scenario. The increased LoS consequentially reduced exhaust emissions on the overall observed motorway segment. The simultaneous improvement of TTS and TT and the reduction of vehicle emissions due to the application of VSLC highlight the significance of optimal traffic control on existing road infrastructure to achieve EU goals for a greener transport environment. The results obtained were correlated with the strength of the controller's action. The controller influences the dynamics of traffic flow and this influence is determined by an appropriate value of the controller gain parameter $K_{\mathrm{v}}$. In line with that, the positive effect of both controllers analysed was found to depend on their value of $K_{\mathrm{v}}$. Optimal $K_{\mathrm{v}}$ values were determined for the simulated urban motorway segment.

The results obtained in this study directly confirm that VSLC with appropriately chosen control parameters can alleviate traffic congestion and improve the LoS of urban motorways under the assumption, of course, that drivers actually comply with the imposed speed limit. Different compliance rates of drivers to imposed speed limits can affect the efficiency of VSLC, which is a topic that requires attention in future work.

According to EU plans to significantly reduce GHG emissions produced by the EU transport sector to minimise the negative impact on the environment, the reduction of exhaust emissions by applying VSLC shows potential for application on urban motorways. However, further analysis of this is needed as VSLC can be applied in co-operation with other traffic-control approaches such as ramp metering. Such co-operation could potentially further improve the LoS on urban motorways, including reductions of exhaust emissions. Different control approaches for improving the efficiency of VSLC and its application on EU urban motorways should also be examined to contribute to further reductions in exhaust emissions. This is especially true as, in the future environment of connected and autonomous vehicles, a higher compliance rate to posted speed limits can be achieved.

\section{Acknowledgements}

The authors thank PTV Group for providing student licences for the microscopic simulator Vissim and the vehicle emissions simulator Enviver. Thanks also go to Nino Korent for help with implementation of the MVM controller. This research was carried out within the activities of the Centre of Research Excellence for Data Science and Cooperative Systems supported by the Ministry of Science and Education of the Republic of Croatia. This work was funded by the Faculty of Transport and Traffic Sciences, University of Zagreb, in the scope of the PROM-PRO project 'Navigation of electric vehicles with the criteria of minimal energy consumption' (grant number 998-9).

\section{REFERENCES}

Brandt R, Borchiellini R, Dandie B et al. (2012) Road Tunnels: Vehicle Emissions and Air Demand for Ventilation. World Road Association (PIARC), Paris, France, Technical Report 2012R05EN.

Carlson RC, Papamichail I and Papageorgiou M (2010) Local feedback-based mainstream traffic flow control on freeways using variable speed limits. Proceedings of the 13th International IEEE Annual Conference on Intelligent Transportation Systems, Madeira Island, Portugal, pp. 513-518.

Dervisoglu G, Gomes G, Kwon J et al. (2009) Automatic calibration of the fundamental diagram and empirical observations on capacity. Proceedings of the Transportation Research Board 88th Annual Meeting, Washington, DC, USA.

EC (European Commission) (2011) White Paper: Roadmap to a Single European Transport Area - Towards a Competitive and Resource Efficient Transport System. EC, Brussels, Belgium. 
EEA (European Environment Agency) (2018) Greenhouse Gas Emissions From Transport in Europe. EEA, Copenhagen, Denmark. See https://www.eea.europa.eu/data-and-maps/indicators/transportemissions-of-greenhouse-gases/transport-emissions-of-greenhousegases-11 (accessed 03/10/2019)

Falcocchio JC and Levinson HS (2015) Road Traffic Congestion: A Concise Guide (Springer Tracts on Transportation and Traffic). Springer International, Cham, Switzerland.

Grabec I (2014) Forecasting of traffic jams at disturbed sections of high-ways. Journal of Applied \& Computational Mathematics 3(4), pp. 168-176, https://doi.org/10.4172/2168-9679.1000168.

Gregurić M, Ivanjko E, Korent N and Kušić K (2016) Short review of approaches for variable speed limit control. Proceedings of International Scientific Conference on Science and Transport Development (ZIRP 2016), Zagreb, Croatia, pp. 41-52.

Hadjipollas M, Lestas M, loannou O, Hadjipollas G and Pitsillides A (2008) Evaluation of new ramp metering and variable speed limit algorithms on the Cyprus highway network. Proceedings of International Trade and Freight Transportation Conference (ITFTC2008), Ayia Napa, Cyprus, pp. 171-177.

Higgs B, Abbas M and Medina A (2011) Analysis of the Wiedemann car following model over different speeds using naturalistic data Proceedings of the 3rd International Conference on Road Safety and Simulation, Indianapolis, IN, USA.

Khondaker B and Kattan L (2015) Variable speed limit: an overview. Transportation Letters 7(5): 264-278.

Kušić K, Korent N, Gregurić M and Ivanjko E (2016) Comparison of two controllers for variable speed limit control. Proceedings of the 58th International Symposium ELMAR-2016, Special session Intelligent Transport Systems, Zadar, Croatia, pp. 101-106.

Leyn U and Vortisch P (2015) Calibrating Vissim for the German highway capacity manual. Transportation Research Record $\mathbf{2 4 8 3}$ 74-79, https://doi.org/10.3141/2483-09.

Li D and Ranjitkar P (2015) A fuzzy logic-based variable speed limit controller. Journal of Advanced Transportation 49(8): 913-927, https://doi.org/10.1002/atr.1320.

Müller ER, Carlson RC, Kraus W and Papageorgiou M (2015) Microsimulation analysis of practical aspects of traffic control with variable speed limits. IEEE Transactions on Intelligent Transportation Systems 16(1): 512-523, https:/doi.org/10.1109/ TITS.2014.2374167.

Papageorgiou M, Hadj-Salem H and Blosseville JM (1991) ALINEA: a local feedback control law for on-ramp metering. Transportation Research Record 1320: 58-64.

Papageorgiou M, Kosmatopoulos E and Papamichail I (2008) Effects of variable speed limits on motorway traffic flow. Transportation Research Record 2047: 37-48, https:/doi.org/ 10.3141/2047-05

PTV Group (2019) PTV Vissim Simulation Software. PTV Group, Karlsruhe, Germany. See http://vision-traffic.ptvgroup.com (accessed 02/05/2019).

Rämä P and Schirokoff A (2004) Effects of weather-controlled variable speed limits on injury accidents. Proceedings of the 4th European Congress on Intelligent Transportation Systems and Services, Budapest, Hungary.

Roess RP, Prassas ES and McShane WR (2011) Traffic Engineering, 4th edn. Prentice Hall, Upper Saddle River, NJ, USA.

Rosas-Jaimes OA, Téllez LAQ and Anaya GF (2016) Polynomial approach and non-linear analysis for a traffic fundamental diagram. PROMET - Traffic Transportation 28(4): 321-329, https://doi.org/10.7307/ptt.v28i4.1965.

Sadat M and Celikoglu HB (2017) Simulation-based variable speed limit systems modelling: an overview and a case study on Istanbul freeways. Transportation Research Procedia 22: 607-614, https://doi.org/10.1016/j.trpro.2017.03.051

Smaragdis E and Papageorgiou M (2003) Series of new local ramp metering strategies. Transportation Research Record 1856: 74-86, https://doi.org/10.3141/1856-08.

TNO (Netherlands Organisation for Applied Scientific Research) (2019) Enviver Simulation Software. TNO, Amsterdam, the Netherlands. See http://www.tno.nl (accessed 05/02/2019).

TRB (Transportation Research Board) (2000) Highway Capacity Manual. TRB, National Research Council, Washington, DC, USA.

Wang Y (2011) Dynamic Variable Speed Limit Control: Design, Analysis and Benefits. PhD thesis, University of Southern California, Los Angeles, CA, USA. 